\title{
A COLANGIOPANCREATOGRAFIA POR RESSONÂNCIA MAGNÉTICA DEVE SUBSTITUIR A COLANGIOPANCREATOGRAFIA ENDOSCÓPICA RETRÓGRADA NO ALGORITMO DIAGNÓSTICO DAS DOENÇAS BILIOPANCREÁTICAS?
}

\author{
DESCRITORES - Imagem por ressonância magnética. Pancreatocolangiografia retrógrada endoscópica.
}

Pancreatopatias, diagnóstico. Doenças das vias biliares, diagnóstico.

A canulação endoscópica da papila duodenal maior, seguida da injeção do meio de contraste e obtenção de colangiopancreatografia, foi sistematizada primeiramente por OI, em $1970^{(10)}$.

Desde então, a colangiopancreatografia endoscópica retrógrada (CPER) assumiu papel definitivo no algoritmo de investigação da síndrome de icterícia colestática ${ }^{(12)}$, especialmente aquela associada à dilatação das vias biliares. Tal fato se deve à acurácia inigualável do método em demonstrar a presença de cálculos, estenoses e más-formações biliopancreáticas, além de fornecer opção de tratamento.

Contudo, apesar da difusão do método, do seu ensino em meios acadêmicos, da melhoria dos equipamentos, acessórios e dos cuidados no preparo e seguimento dos pacientes, a CPER apresenta, mesmo em mãos experientes, morbidade que pode atingir $6 \%{ }^{(5)}$. Basicamente a pancreatite aguda é a complicação mais freqüente e temida da CPER diagnóstica. Relaciona-se, em geral, à dificuldade na canulação seletiva da via biliar, resultando em seguidas injeções de contraste no sistema ductal pancreático ou, ainda, na aplicação do "pré-corte" que, definitivamente, acarreta risco de pancreatite, perfuração e hemorragia ${ }^{(2)}$. Certamente, muitos daqueles que realizam rotineiramente a CPER já vivenciaram casos de pancreatite aguda de intensidade variável, advinda desse exame, onde a via biliar provou ser de difícil canulação, suscitando a realização de "pré-corte" que, bem sucedido, permitiu a obtenção do colangiograma normal.

TRAPP et al. ${ }^{(14)}$ analisaram 39 processos judiciais movidos por pacientes e familiares, devido à ocorrência de pancreatite aguda pós-CPER diagnóstica, em sua maioria, no período de 1992 a 1996. Apesar da indicação correta do exame, na maior parte dos casos (31/39), as ações foram julgadas procedentes em quase $80 \%$ das vezes (30/39). O "pré-corte" foi utilizado em sete ocasiões (18\%). Pancreatite grave e fatal ocorreram, respectivamente, em 24 (62\%) e 9 casos (23\%). Esse estudo torna óbvia a necessidade de método de igual acurácia e menor morbidade em relação à CPER, que deixaria de ocupar o lugar de destaque em que esteve por tantos anos no algoritmo diagnóstico da icterícia obstrutiva. Neste cenário, as conseqüências dos fatos acima seriam mais profundas. A indicação da CPER, com fins puramente diagnósticos, poderia se constituir até mesmo em má prática médica, exceção feita a situações especiais.

O método que pretende substituir a CPER, além de menos invasivo e bastante sensível, deve ser específico, uma vez que, não raramente, lesões benignas e malignas fazem parte 
Maluf-Filho F. A colangiopancreatografia por ressonância magnética deve substituir a colangiopancreatografia endoscópica retrógrada no algoritmo diagnóstico das doenças biliopancreáticas?

do diagnóstico diferencial da icterícia obstrutiva, fazendo com que a conduta possa variar desde papilotomia endoscópica, até procedimentos ressectivos de maior envergadura, como a gastroduodenopancreatectomia ${ }^{(7)}$.

A colangiopancreatografia por ressonância magnética (CPRM) baseia-se no princípio físico do sinal hiperintenso dos fluídos estacionários (bile, derrames cavitários, cistos) em T2, descrito por WALLNER et al. ${ }^{(15)}$, em 1991. Assim, sem a injeção de contraste ou de radiação ionizante, são obtidas imagens-fonte, de cortes axiais, das vias biliares e pancreáticas. Posteriormente, tais imagens são reconstruídas no plano coronal, utilizando "software" específico, resultando nas imagens a que nos acostumamos a apreciar nos últimos anos.

No estudo, atual e de grande interesse prático, publicado nesta edição dos ARQUIVOS de GASTROENTEROLOGIA, PISANI et al. ${ }^{(11)}$ compararam a CPRM e a CPER em 41 pacientes com diagnóstico sindrômico de icterícia colestática. Destacam-se alguns pontos positivos da investigação: estudo prospectivo em centro com reconhecida experiência em CPER; todos os exames de CPER foram feitos por único examinador; todos os exames de CPRM foram interpretados por um único examinador; o intervalo de tempo entre a realização dos dois exames foi inferior a $24 \mathrm{~h}$; o examinador da CPER desconhecia os resultados da CPRM e vice-versa. Os fatos acima reforçam a confiabilidade nos resultados obtidos.

Dos 41 pacientes, $6(15 \%)$ não apresentaram lesão biliopancreática. Tal situação foi avaliada adequadamente pela CPER em todos os casos e, em 4, pela CPRM. As causas de falha desta última foram aerobilia pós-papilotomia, confundida com cálculo biliar; na outra falha, a CPRM sugeriu a presença de pancreas divisum, não confirmado à CPER. Estas duas falhas comprometeram a sensibilidade do método em identificar a via biliopancreática normal. Ou ainda, resultaram na baixa especificidade, quando o método apontou para doença naquelas vias. Tais achados, se reproduzidos em casuísticas maiores, descredenciariam a CPRM como método de diagnóstico de rastreamento ou de primeira intenção, pois o achado de anormalidade poderia esconder, de fato, paciente "normal", que seria, desnecessariamente, submetido a intervenção endoscópica, percutânea ou cirúrgica. Como os próprios autores admitem, este achado não ocorre em outras séries. Ao contrário, SOTO et al. ${ }^{(13)}$, realizando protocolo semelhante, identificaram 16 dos 17 pacientes com via biliar normal (especificidade de 94,1\%), numa coorte de 46 pacientes com icterícia obstrutiva. No ano seguinte, HINTZE et al. ${ }^{(3)}$ publicaram série maior, com 78 pacientes, obtendo resultados semelhantes. Em 1998, ADAMEK et al. ${ }^{(1)}$ relataram especificidade de $100 \%$ à CPRM no diagnóstico diferencial da icterícia obstrutiva (via biliar normal $\mathrm{x}$ via biliar alterada) em 60 pacientes, credenciando o método a evitar exames desnecessários de CPER.

No estudo de PISANI et al. ${ }^{(11)}$, a CPRM apresentou a mesma acurácia da CPER no diagnóstico dos cálculos biliares, dado este amplamente comprovado na literatura ${ }^{(1,3,13)}$. De fato, em algumas situações, os métodos poderiam ser considerados complementares. Na suspeita de litíase intra-hepática, sugere-se que a CPRM possa ter resultados melhores do que a CPER. Ao contrário, na situação pós-papilotomia em que há aerobilia, a CPER é superior, pois a dúvida diagnóstica entre cálculo e ar é dirimida através da instrumentação direta da via biliar. Assim, em paciente com papilotomia prévia e sinais de aerobilia difusa, o exame diagnóstico de eleição para detecção da litíase biliar é, a princípio, a própria CPER.

Outras limitações da CPRM são pequenos cálculos biliares, menores de $4 \mathrm{~mm}$ e as pequenas lesões expansivas periampulares. Neste sentido, destaca-se a acurácia da ecoendoscopia (EE) na detecção de tumores pancreáticos e ampulares menores de $20 \mathrm{~mm}$ e de cálculos menores de $5 \mathrm{~mm}$. De fato, MATERNE et al. ${ }^{(9)}$ compararam a CPRM e a EE na detecção de causas da obstrução da via biliar extra-hepática, em 50 pacientes. As acurácias diagnósticas foram, respectivamente, $92 \%$ e $94 \%$.

$\mathrm{Na}$ era da cirurgia vídeo-assistida, houve crescimento do interesse no diagnóstico pré-operatório da coledocolitíase, uma vez que a exploração laparoscópica do colédoco é complexa e onerosa. Por outro lado, nesta situação, não se deseja "converter" o procedimento, perdendo as vantagens do acesso laparoscópico. Neste cenário, apenas os pacientes de altíssimo risco para coledocolitíase (icterícia, colangite, cálculos de colédoco à ecografia convencional) deveriam ser enviados à CPER pré-operatória. Aqueles pacientes de riscos alto e intermediário (dilatação da via biliar principal e/ou alterações de enzimas hepática), em que a chance de coledocolitíase vai de $10 \%$ a $30 \%$, se beneficiariam de testes menos invasivos, tais como a CPRM e a EE, a depender da disponibilidade e experiência e custos locais. Finalmente, aqueles de baixo risco, seriam submetidos a colangiografia intra-opeatória. LIU et al. ${ }^{(4)}$, utilizando algoritmo semelhante, aplicou a CPER em 27 de 440 pacientes (6\%), encontrando coledocolitíase em 25 deles $(93 \%)$. Por outro lado, indicou a CPRM para 37 pacientes (8\%), encontrando coledocolitíase em $32 \%$ deles. Apenas $4 \%$ dos 376 pacientes não submetidos a CPER ou a CPRM apresentaram coledocolitíase no estudo intra-operatório. A melhoria da relação custo-benefício e da qualidade assistencial deste algoritmo parece óbvia e merece, no mínimo, ser validada localmente.

No artigo em questão ${ }^{(11)}$, a CPRM identificou corretamente a estenose biliar em $89 \%$ dos casos. A acurácia da CPRM na detecção de estenoses biliares benignas pós-operatórias, pós-transplante hepático ou daquelas malignas, está bem documentada ${ }^{(1,3,13)}$. Neste sentido, não se deve esquecer de que a CPRM é derivada de ressonância magnética. Assim, a pedido do médico-assistente, é possível realizar ressonância magnética, angioressonância, e CPRM, obtendo-se informações sobre o tumor, a presença de linfonodos e de extensão para grandes vasos. Nestes parâmetros, a CPRM e a tomografia helicoidal (TCH) parecem ser equivalentes. Por outro lado, a CPER ficaria reservada para procedimentos terapêuticos, quando não se 
prevê tratamento cirúrgico para estes pacientes, ou quando a drenagem biliar pré-operatória se impõe (insuficiência renal, coagulopatia, colangite). Outra situação em que a CPER ficaria indicada é quando da necessidade de se obter material para análise histológica. A associação de escovado e biopsia de estenose biliar maligna tem acurácia diagnóstica de $75 \%$. Nos tumores pancreáticos, o método de escolha para obtenção de material é a punção guiada por ecoendoscopia, dada sua elevada eficácia (85\%), morbidade e risco de colangite praticamente nulos.

Mesmo em situações em que a CPER está indicada com fins de drenagem paliativa da via biliar, a CPRM pode ser útil. O principal exemplo consiste no colangiocarcinoma da confluência dos hepáticos (tumor de Klatskin), em que a drenagem endoscópica é tecnicamente difícil, não raramente incompleta, levando à colangite de difícil tratamento. Há fortes indícios de que, nestes casos, a preferência seja drenar o segmento que foi contrastado durante o exame colangiográfico. Assim, através de CPRM, ZIDI et al. ${ }^{(16)}$ classificaram corretamente $16(80 \%)$ dos 20 colangiocarcinomas hilares, conforme a classificação de Bismuth. A CPER foi realizada sem o conhecimento prévio da CPRM. Seis pacientes (30\%) foram drenados inadequadamente e evoluíram com colangite. Utilizando-se deste princípio, HINTZE et al. ${ }^{(3)}$ orientaram a drenagem biliar por via endoscópica, baseando-se nos achados de CPRM, em 35 pacientes. Procuraram contrastar e drenar um único lobo. A resolução da icterícia se deu em $86 \%$ dos pacientes. Apenas $6 \%$ evoluíram com colangite. Estes dados justificam, no mínimo, a realização de estudos comparativos.

Vale ressaltar que a acurácia da ressonância para o estudo das via biliares não se repete para os ductos pancreáticos. Assim, novos métodos, tais como a pancreatorressonância dinâmica, feita com injeção de secretina, melhora a visibilização dos ductos pancreáticos ${ }^{(6)}$.

Por fim, qual a melhor resposta para a pergunta que serve de título para este editorial? Provavelmente, um "tímido" sim. Para o diagnóstico da litíase e da estenose biliar, a CPRM tem condições de, a grosso modo, substituir a CPER. O estudo ora apresentado corrobora esta idéia. Além de todas as exceções e restrições, mais alguns detalhes merecem atenção. Quando da suspeita de obstáculo biliar extrahepático, a EE é ainda método superior. Quando da impossibilidade da realização da CPRM, a TCH é método acurado e deve ser empregado.

Todos os dados aqui discutidos se baseiam em CPRM realizadas em centros de excelência. Assim, destaca-se a iniciativa do grupo liderado por PISANI ${ }^{(11)}$, que validou localmente a acurácia da CPRM frente à CPER. Assim, poderão indicar a seqüência mais adequada de exames, pois sabem das limitações e da eficácia de cada um. Este é exemplo a ser seguido pelos centros de gastroenterologia.

Por fim, nenhum teste diagnóstico deve ser analisado independentemente do quadro clínico. O raciocínio clínico adequadamente fundamentado continua a ser a coluna mestra de qualquer algoritmo diagnóstico.

Fauze MALUF-FILHO*

Maluf-Filho F. Should magnetic resonance cholangiopancreatography replace endoscopic retrograde cholangiopancreatography for diagnostic purposes? Arq Gastroenterol 2001;38(3):145-148.

HEADINGS - Magnetic resonance imaging. Cholangiopancreatography, endoscopic retrograde. Pancreatic diseases, diagnosis. Bile duct diseases, diagnosis.

\section{REFERÊNCIAS BIBLIOGRÁFICAS}

1. Adamek HE, Albert J, Weitz M, Breer H, Schilling D, Riemann JF. A prospective evaluation of magnetic resonance cholangiopancreatography in patients with suspected bile duct obstruction. Gut 1998;43:680-3

2. Cotton PB. Precut papilotomy: a risky technique for experts only. Gastrointest Endosc 1983;5:57-9.

3. Hintze RE, Adler A, Veltzke W, Abou-Rebyeh H, Hammerstingl R, Vogl T, Felix R. Clinical significance of magnetic resonance cholangiopancreatography (MRCP) compared to endoscopic retrograde cholangiopancreatography (ERCP). Endoscopy 1997;29:182-7.
4. Liu TH, Consorti ET, Kawashima A, Tamm EP, Kwong KL, Gill BS, Sellin JH, Peden EK, Mercer DW. Patient evaluation and management with selective use of magnetic resonance cholangiopancreatography and endoscopic retrograde cholangiopancreatography before laparoscopic cholecystectomy. Ann Surg 2001;234:33-40.

5. Loperfido S, Angelini G, Benedetti G, Chilovi F, Constan F, De Bernanrdinis F, De Bernardinis M, Ederle A, Fina P, Fratton A. Major early complications from diagnostic and therapeutic ERCP: a prospective multicenter study. Gastrointest Endosc 1998;48:1-10

6. Magnetic resonance cholangiopancreatography-guided unilateral endoscopic stent placement for Klatskin tumors. Gastrointest Endosc $2001 ; 53: 40-6$.

* Doutor pelo Departamento de Gastroenterologia da Faculdade de Medicina da Universidade de São Paulo. 
Maluf-Filho F. A colangiopancreatografia por ressonância magnética deve substituir a colangiopancreatografia endoscópica retrógrada no algoritmo diagnóstico das doenças biliopancreáticas?

7. Maluf-Filho F. Contribuição da ecoendoscopia à avaliação das neoplasias malignas do pâncreas e da papila duodenal maior [tese de doutorado]. São Paulo: Faculdade de Medicina da Universidade de São Paulo; 2000.

8. Manfredi R, Costamagna G, Brizi MG, Maresca G, Vecchioli A, Colagrande C, Marano P. Severe chronic pancreatitis versus suspected pancreatic disease: dynamic MR cholangiopancreatography after secretin stimulation. Radiology 2000;214:849-55.

9. Materne R, Van Beers E, Gigot JF, Jamart J, Geubel A, Pringot J, Deprez P. Extrahepatic biliary obstructions: magnetic resonance imaging compared with endoscopic ultrasonography. Endoscopy 2000;32:3-9.

10. Oi I. Fiberduodenoscopy and endoscopic pancreatocholangiography. Gastrointest Endosc 1970;17:59-62.

11. Pisani JC, Bacelar A, Malafaia O, Ribas-Filho JM, Czezcko NG, Nassif PAN. Estudo comparativo entre a colangiopancreatografia por ressonância magnética e a colangiopancreatografia endoscópica no diagnóstico das obstruções biliopancreáticas. Arq Gastroenterol 2001;38:149-57.
12. Sherlock S, Dooley J. Jaundice. In: Sherlock S, Dooley J, editors. Diseases of the liver and biliary system. 10.ed. Oxford: Blackwell Science; 1997. p.20115.

13. Soto JA, Barish MA, Yucel EK, Siegenberg D, Ferrucci JT, Chuttani R. Magnetic resonance cholangiography: comparison with endoscopic retrograde cholangiopancreatography. Gastroenterology 1996;110:589-97.

14. Trap R, Adamsen S, Hart-Hansen O, Henriksen M. Severe and fatal complications after diagnostic and therapeutic ERCP: a prospective series of claims to insurance covering public hospitals. Endoscopy 1999;31:125-30.

15. Wallner BK, Schumacher KA, Weidenmaier W. Dilated biliary tract: evaluation with MR cholangiography with a T2-weighted contrast-enhanced fast sequence. Radiology 1991;181:805-8.

16. Zidi SH, Prat F, Le Guen O, Rondeau Y, Pelletier G. Performance characteristics of magnetic resonance cholangiography in the staging of malignant hilar strictures. Gut 2000;46:103-6. 